\title{
Prospects for parity-nonconservation experiments with highly charged heavy ions
}

\author{
M. Maul, A. Schäfer, and W. Greiner \\ Institut für Theoretische Physik, Johann Wolfgang Goethe-Universität, D-60054 Frankfurt am Main, Germany \\ P. Indelicato \\ Laboratoire de Physique Atomique et Nucléaire, CNRS URA No. 771, Université Pierre et Marie Curie, \\ Case 93, 4 place Jussieu, F-75252 Paris Cedex 05, France
}

(Received 30 October 1995)

\begin{abstract}
We discuss the prospects for parity-nonconservation experiments with highly charged heavy ions. Energy levels and parity mixing for heavy ions with 2-5 electrons are calculated. We investigate two-photon transitions and the possibility of observing interference effects between weak-matrix elements and Stark matrix elements for periodic electric field configurations. [S1050-2947(96)08905-6]

PACS number(s): 32.30. $-\mathrm{r}, 32.90 .+\mathrm{a}$
\end{abstract}

\section{INTRODUCTION}

Atomic physics tests of the standard model [1-3] play a very special role because of the small momentum transfers involved. Comparisons between their results and high energy data are highly sensitive to radiative corrections and thus to extensions of the standard model [4]. With the percent precision reached in the Cs experiments described in [3], the effect of radiative corrections is of the order of the experimental accuracy. If a system is found for which a $0.1 \%$ accuracy can be reached, the experimental results would allow most interesting and far reaching conclusions (see, e.g., [5]). For the atoms and experimental setups studied so far, this unfortunately seems to be out of reach, which motivates the search for significantly different alternatives. The possibility we want to discuss is the use of highly charged heavy ions, which can be produced and stored in great variety at, e.g., Gesellschaft für Schwerionenforschung in Darmstadt, Germany. We already discussed some time ago the prospects for inducing a two-photon transition in heliumlike uranium [6]. In this paper we extend our studies to systems with up to five electrons and we adopt the ingenious ideas proposed by Botz, Bruß, and Nachtmann [7] especially suited to the investigation of parity-violating effects in storage rings.

The starting point for all such experiments is that, due to the parity-violating exchange of neutral $Z$ bosons between nucleus and electrons, every electron state is mixed with states of opposite parity. In first order perturbation theory the coefficient $\eta$ of this admixture is given by

$$
\eta=\frac{\left\langle i\left|\frac{G_{F}}{2 \sqrt{2}}\left(1-4 \sin ^{2} \vartheta_{\mathrm{W}}-\frac{N}{Z}\right) \rho \gamma_{5}\right| f\right\rangle}{E_{i}-E_{f}},
$$

where $G_{F}$ denotes Fermi's constant, $\vartheta_{W}$ the Weinberg angle, $N$ the neutron number, $Z$ the proton number, and $\rho$ the nuclear density normalized to $Z$. From this formula we see why heavy ions with few electrons left in inner shells are good candidates for investigating parity-nonconservation effects: The admixture coefficient $\eta$ is very large (typically orders of magnitude larger than for usual, neutral atoms) due to the big overlap between the nucleus and the electron states. The other factor that can make $\eta$ large is the energy difference between the two mixing electronic states $i$ and $f$ that ought to be very small. Therefore, we are especially interested in level crossings of electron states with the same spin but opposite parity.

It was pointed out in [4] that Eq. (1) has to be modified by radiative corrections, the weak charge $Q_{W}$ included in (1) changes according to

$$
Q_{W}=Z-4 Z \sin ^{2} \vartheta_{\mathrm{W}}-N \rightarrow \rho_{P V}^{\prime}\left(Z-4 Z \kappa_{P V}^{\prime} \sin ^{2} \vartheta_{\mathrm{W}}-N\right) .
$$

Here $\rho_{P V}^{\prime}$ and $\kappa_{P V}^{\prime}$ are constants that arise from the radiative corrections mentioned above. The crucial point is that they depend on the masses of the particles involved in the radiative processes, especially the top quark and the Higgs boson. Since it seems now that there is evidence for the top quark to exist, it should, from a theoretical point of view, be possible to determine from $\rho_{P V}^{\prime}$ and $\kappa_{P V}^{\prime}$ the value of the mass of the Higgs boson that makes the standard model renormalizable, thus giving important guidance to identify this particle in high energy experiments.

In Sec. II, we will discuss uranium with 2-5 electrons as a model for other heavy ions reaching from gold to plutonium. In Sec. III, we will discuss the possibility of level crossing in compound heavy ions, and, finally, in Sec. IV, we will investigate the possibility of polarization rotations in heavy ions.

\section{HEAVY IONS WITH 2-5 ELECTRONS IN INNER SHELLS}

For an experiment with heavy ions with few inner shell electrons, we have to give a criterion by which we can judge the feasibility of such an experiment. As such a criterion, we should compare the $\eta$ values of the systems regarded here with the $\eta$ value of the heliumlike uranium system discussed in [6], i.e., $\eta \approx 10^{-6}$, when taking the energy difference to $\Delta E=1 \mathrm{eV}$. Even this relatively high value of $\eta$ left the proposed experiment beyond the scope of experimental feasibility for the setup discussed there. 
The following consideration leads to a second restriction. If, for example, the electron states of interest are excited during the stripping process of the ion in a stripping foil, then for any realistic experiment the experimental setup should be placed a little distance behind this foil, let's say one meter. Then the lifetime of these excited states should be long enough to survive this one meter of flight. Taking into account a time dilation factor of about 5 for an ion accelerated to $5 \mathrm{GeV}$ per nucleon, the lifetime should be larger than $\approx 10^{-9} \mathrm{~s}$. This would be an optimal value, but a lifetime of $10^{-10} \mathrm{~s}$, corresponding to a distance of $10 \mathrm{~cm}$, would probably do also.

We furthermore consider only the lowest lying electron states that offer a possibility for a parity-violation experiment. Since the parity admixture is proportional to the overlap of the electron states in question with the nucleus, this admixture should become very large for low lying states if the energies are sufficiently degenerate.

We should state that these criteria do not rule out all imaginable experiments. It could be possible, for example, to store ions in an ion trap and to generate the excited state by a laser beam, perhaps by a laser that has yet to be invented or that will be available in a few years; the question of the lifetime of the electron states may then be superfluous. Also, there could be other electronic configurations in the ions studied here or in ions having a few more electrons, with suitable properties. The relativistic corrections can lead to very rich structures, with level crossings and metastable states that have just begun to be explored (see, for example, $[8,9])$ and can lead to increased sensitivity to, e.g., electric quadrupole hyperfine interaction [10].

The systems we are interested in are highly charged heavy ions for which two states with equal angular momentum $J$ but opposite parity have similar energy. We have investigated the binding energies of the lower lying levels of ions with 2-5 electrons to identify the most promising candidates. When not available from the literature, energies were calculated with the multiconfiguration Dirac-Fock Program (MCDF) published by Grant et al. [11], which provide a relativistic correction as well as one-electron QED corrections and approximate, although inaccurate, many-body QED corrections. For heliumlike systems, however, we can use very precise MCDF or relativistic configurationinteraction (RCI) calculations including correlation and QED effects.

For each electron configuration, we show as an example the results for uranium. There are no noticeable qualitative differences for other heavy ions down to gold, as is graphically shown for the electron states of interest, except for the two-electron $1 s 2 s{ }^{1} S_{0} \rightarrow 1 s 2 p{ }^{3} P_{0}$ case for which two crossings at $Z \approx 62$ and $Z \approx 92$ occur. Since in this section we do only exploratory work, we do not claim a precision much better than a few eV, except for two-electron systems. Lifetimes are calculated in the LS configuration from elementary atomic physics. We take the inverse of the main transition probability to be the lifetime of the respective state, neglecting thereby other contributions of lower order. The parity admixture coefficient in this second section is determined only for the main electron state and therefore also gives only the order of magnitude.

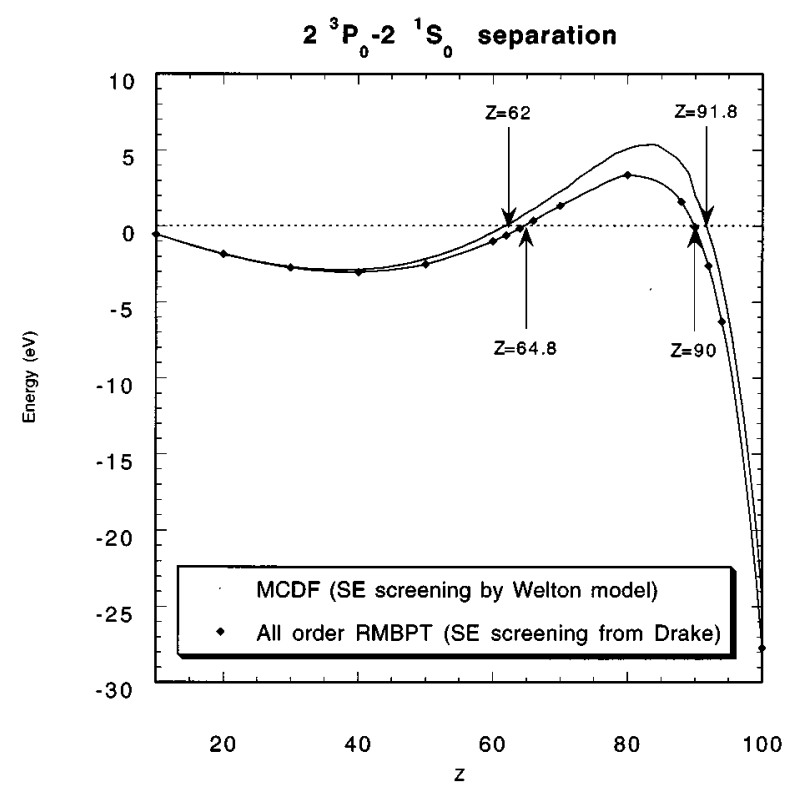

FIG. 1. Energy difference between the two nearly degenerated electron states as a function of atomic number.

\section{A. Two-electron ions}

Extensive calculations of the binding energies of twoelectron ions have appeared in the literature over the past ten years [12-14]. In Fig. 1, we plot the $1 s 2 s{ }^{1} S_{0} \rightarrow 1 s 2 p^{3} P_{0}$ energy difference as calculated in [12] and [15]. The first one is an all-order relativistic many-body perturbation theory (RMBPT) calculation, which uses the two-body QED corrections of Ref. [14]. The second calculation is a MCDF calculation done along the lines of $[13,16]$, which uses the Welton model for two-body self-energy corrections, experimental nuclear size when available, and includes a finite-nuclear size correction to the self-energy [17]. The energy separation between $1 s 2 s{ }^{1} S_{0}$ and $1 s 2 p{ }^{3} P_{0}$ is plotted in Fig. 1 as a function of $Z$. In order to show how this level crossing happens, we show in detail the contributions to the energy separation at $Z=62$ and 92 in Table I. It should be noted that this crossing mostly involves the interplay between magnetic energy and QED correction contributions.

With this new energy determination, the parity admixture $|\eta| \approx 5 \times 10^{-6} \mathrm{eV} /(\Delta E)[6]$ would be enhanced by a factor of 3. For the experimental setup discussed in [6] with the detection of a laser-induced two-photon transition, the laser intensity required would still be unrealistically large, of order $10^{21} \mathrm{~W} / \mathrm{cm}^{2}$ (presently, only lasers up to an intensity of $10^{17}-10^{18} \mathrm{~W} / \mathrm{cm}^{2}$ exist). The main problem in this context is that the heavy ions are only available in the form of a rapid ion beam and that the only possibility of exciting the electron states of interest is by means of the stripping process.

One hope for improving the situation is to study different isotopes to see if one can still reduce the energy difference. Figure 2 shows that by choosing suitable isotopes, the degeneracy can be improved. Only the Coulomb energy is modified due to the change in nuclear radius. For uranium, the energy separation does cancel between isotopes 233 and 234, within the present calculation. One should keep in mind, 
TABLE I. Contributions to the $1 s 2 s{ }^{1} S_{0} \rightarrow 1 s 2 p{ }^{3} P_{0}$ separation near the two crossing points. All units are given in $\mathrm{eV}$.

\begin{tabular}{lcccccc}
\hline \hline & $Z=62$ & & & $Z=92$ \\
& $1 s 2 p{ }^{3} P_{0}$ & $1 s 2 s{ }^{1} S_{0}$ & Diff. & $1 s 2 p{ }^{3} P_{0}$ & $1 s 2 s{ }^{1} S_{0}$ & Diff. \\
\hline Coulomb & -68868.56 & -68861.61 & -6.948 & -165518.05 & -165487.55 & -30.50 \\
Magnetic & 38.30 & 17.12 & 21.17 & 151.30 & 66.36 & 84.91 \\
Retardation & -3.26 & 1.30 & -4.56 & -10.09 & 5.56 & -15.65 \\
Mass pol. & -0.029 & 0.00 & -0.03 & -0.04 & 0.00 & -0.04 \\
Correlation & -0.39 & -0.59 & 0.20 & -1.02 & -1.18 & 0.16 \\
le self-energ. & 82.66 & 95.16 & -12.50 & 364.88 & 420.68 & -55.80 \\
$2 e$ self-energ. & -0.18 & -1.24 & 1.06 & -1.15 & -5.44 & 4.29 \\
Uehling & -13.52 & -15.18 & 1.67 & -96.13 & -108.71 & 12.59 \\
& -0.02 & -0.10 & 0.087 & -0.28 & -0.89 & 0.61 \\
Wichman and Kroll & 0.38 & 0.42 & -0.04 & 4.75 & 5.28 & -0.53 \\
Kallen and Sabry & -0.11 & -0.12 & 0.01 & -0.73 & -0.83 & 0.09 \\
Nuclear pol. & & & & -1.10 & -1.28 & 0.18 \\
Total energy & -68764.71 & -68764.83 & 0.11 & -165107.70 & -165108.00 & 0.30 \\
\hline \hline
\end{tabular}

however, that the present calculation as well as the one in [12] are not precise enough for finding exactly at which atomic number and for which isotope the crossing occurs. The main uncertainty is in the self-energy screening. In Table I the self-energy screening is evaluated with the Welton model [13], which has been proven to be rather accurate [19], but which is not $a b$ initio. In [12] Drake's screening calculations, which are more adapted to low $Z$, are used. If one uses ab initio QED calculations [18], one gets a larger screening. However [18], did not include relaxation, which seems to be sizable for the $1 s 2 s^{1} S_{0}$ state. For uranium, the Welton model with relaxation gives $4.29 \mathrm{eV}$, while the result from [18] is only $1.08 \mathrm{eV}$. It has been shown on other systems that the Welton model should not be wrong by more than $10 \%$ for this atomic number, while it can be good to $1 \%$ at lower $Z$ [19]. One should note also that higher order radiative corrections (of order $\alpha^{2}$, i.e., of order $\alpha$ with respect to the one-electron self-energy) and QED corrections to the two-photon exchange diagrams [20] have not been evaluated. Both corrections could be as large as $0.5 \mathrm{eV}$. The po-

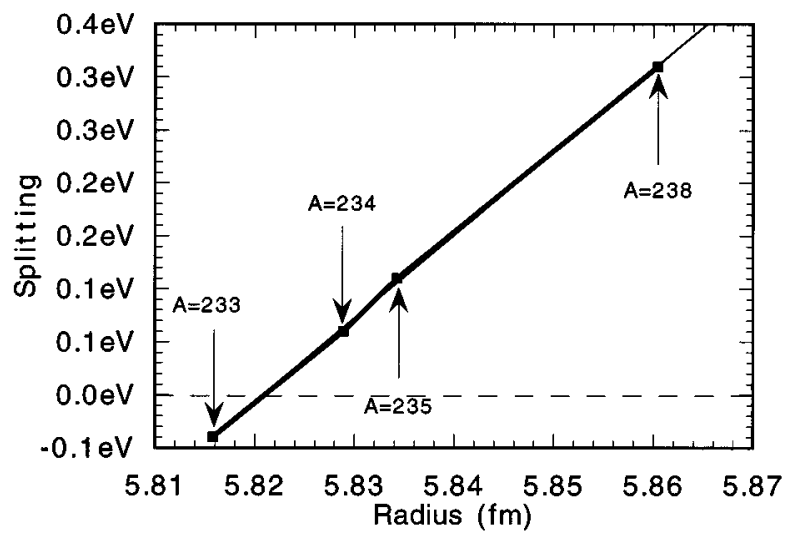

FIG. 2. Energy difference between the two nearly degenerated electron states as a function of the mean-square nuclear radius for $Z=92$. Values of the splitting for experimental nuclear size are represented by squares. sition of the crossing point as well as the smallest energy that can be obtained is thus very uncertain. Also, it should be remembered that if the energy separation is too small it may be difficult to find a laser to excite the two-photon transition.

\section{B. Three- to five-electron ions}

The characteristic feature of the lithiumlike uranium (cf. Table II) is the fact that the ground state and the first excited state already fulfill the main conditions of a parity-violation experiment, i.e., they have the same angular momentum and opposite parity. Moreover, the lifetime of the first excited state lies in the range of $10^{-10} \mathrm{~s}$. Very sophisticated calculations of the ionization energies in lithiumlike uranium, including a discussion of nuclear effects, can be found in [2123]. Complete calculations with relativistic correlation energy and radiative corrections for lower atomic numbers can be found in Refs. [24-26]. Unfortunately, between these two energy states there is a wide energy gap that reduces the magnitude of the parity admixture, which is, in rough approximation, about $\eta=1.4 \times 10^{-8}$. We shall discuss a scheme for detecting parity violation in lithiumlike atoms in Sec. III.

Figure 3 shows that the $Z$ dependence of the energy difference of the first two electron states is nearly linear for atomic numbers in the range $79 \leqslant Z \leqslant 92$, such that no element can be found for which the situation would be substantially different.

The case of berylliumlike ions is comparable to the lithiumlike case. The first two electron levels are, in principle,

TABLE II. Electron configuration of lithiumlike uranium.

\begin{tabular}{lccc}
\hline \hline Main conf. & Parity & Energy (eV) & Lifetime (s) \\
\hline $1 s^{2} 2 s^{2} S_{1 / 2}$ & + & $-2.9424 \times 10^{5}$ & $\infty$ \\
$1 s^{2} 2 p{ }^{2} P_{1 / 2}$ & - & $-2.9395 \times 10^{5}$ & $1.0 \times 10^{-10}$ \\
$1 s^{2} 2 p{ }^{2} P_{3 / 2}$ & - & $-2.8978 \times 10^{5}$ & $1.1 \times 10^{-14}$ \\
$1 s^{2} 3 s^{2} S_{1 / 2}$ & + & $-2.7545 \times 10^{5}$ & $4.9 \times 10^{-15}$ \\
$1 s^{2} 3 p{ }^{2} P_{1 / 2}$ & - & $-2.7537 \times 10^{5}$ & $4.6 \times 10^{-16}$ \\
\hline \hline
\end{tabular}




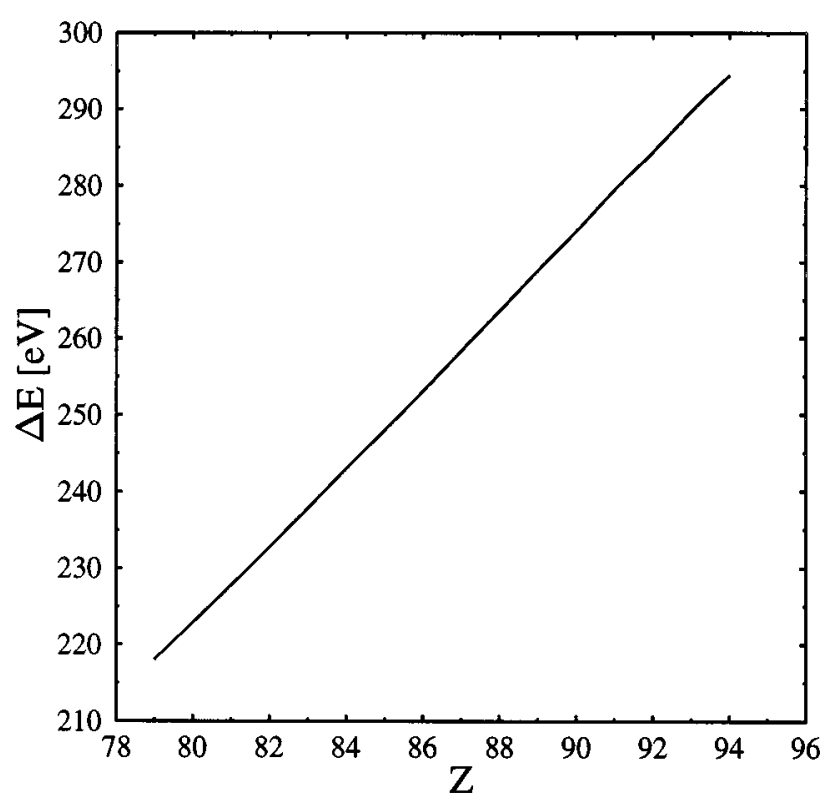

FIG. 3. Energy difference between the first excited state and the ground state in lithiumlike heavy ions from gold to plutonium, $\Delta E=E\left(1 s^{2} 2 p^{2} P_{1 / 2}\right)-E\left(1 s^{2} 2 s^{2} S_{1 / 2}\right)$.

suitable for parity admixture experiments. The lifetime of the first excited state is very large and depends crucially on the spin of the nucleus [27]. In the case of an even-even nucleus, e.g., uranium-238, the lifetime is dominated by a two-photon $E 1 M 1$ transition that is, in general, very slow $\left(10^{7} \mathrm{~s}\right.$ for $Z=82[27]$ ), and can therefore be treated as infinity in comparison with the lifetimes of the next higher levels. In the case of uranium-235, the nucleus has an angular momentum of $7 / 2$ and, due to hyperfine mixing of electron orbitals, the lifetime is severely reduced to $8.562 \times 10^{-5} \mathrm{~s}$ [27].

As a model for berylliumlike heavy ions, we tabulate the energy and lifetime of the lower level of berylliumlike uranium in Table III. In order to achieve reasonable precision, both the ground state and the $1 s^{2} 2 p_{1 / 2}^{2}$ are calculated as the lower and intermediate levels of the $1 s^{2} 2 s^{2}+1 s^{2} 2 p_{1 / 2}^{2}+1 s^{2} 2 p_{3 / 2}^{2} \quad J=0 \quad$ configuration set because intrashell correlation is very large in that case. As in the lithiumlike case, the energy gap between the mixing levels is large, leading to a parity admixture of about $|\eta| \approx 2.4 \times 10^{-8}$.

For a five-electron system, we again examine uranium ions (cf. Table IV). The first two electronic levels are, in principle, usable for a parity-violation experiment, but the comparatively short lifetimes of the first excited state and the

TABLE III. Electron configuration of berylliumlike uranium.

\begin{tabular}{lccc}
\hline \hline Main conf. & Parity & Energy $(\mathrm{eV})$ & Lifetime $(\mathrm{s})$ \\
\hline $1 s^{2} 2 s^{2}{ }^{1} S_{0}$ & + & -326604 & $\infty$ \\
$1 s^{2} 2 s 2 p{ }^{3} P_{0}$ & - & -326345 & $\infty$ for $\mathrm{U}^{238}$ \\
& & & $8.56 \times 10^{-5}$ for $\mathrm{U}^{235}$ \\
$1 s^{2} 2 s 2 p{ }^{3} P_{1}$ & - & -326305 & $1.00 \times 10^{-10}$ \\
$1 s^{2} 2 p^{2}{ }^{3} P_{0}$ & + & -325894 & $7.87 \times 10^{-12}$ \\
$1 s^{2} 2 s 2 p{ }^{3} P_{2}$ & - & -322224 & $3.37 \times 10^{-12}$ \\
\hline \hline
\end{tabular}

TABLE IV. Electron configuration of boronlike uranium.

\begin{tabular}{lccc}
\hline \hline Main conf. & Parity & Energy $(\mathrm{eV})$ & Lifetime $(\mathrm{s})$ \\
\hline $1 s^{2} 2 s^{2} 2 p{ }^{2} P_{1 / 2}$ & - & $-3.5826 \times 10^{5}$ & $\infty$ \\
$1 s^{2} 2 s 2 p^{2}{ }^{4} P_{1 / 2}$ & + & $-3.5785 \times 10^{5}$ & $5.2 \times 10^{-11}$ \\
$1 s^{2} 2 s^{2} 2 p{ }^{2} P_{3 / 2}$ & - & $-3.5417 \times 10^{5}$ & $3.3 \times 10^{-12}$ \\
$1 s^{2} 2 s 2 p^{2}{ }^{4} P_{3 / 2}$ & + & $-3.5389 \times 10^{5}$ & $7.5 \times 10^{-13}$ \\
$1 s^{2} 2 s 2 p^{2}{ }^{2} D_{5 / 2}$ & + & $-3.5384 \times 10^{5}$ & $6.6 \times 10^{-11}$ \\
\hline \hline
\end{tabular}

small admixture of only $|\eta| \approx 9.4 \times 10^{-9}$ make this system completely unattractive. We shall therefore discuss in the following mainly lithiumlike ions.

No level crossing was found for $78 \leqslant Z \leqslant 96$ in any of the three-, four-, and five-electron systems.

\section{LITHIUMLIKE HEAVY IONS WITH HIGH $Z$ AND $N$}

In this section, we study superheavy lithiumlike ions. It is interesting to see how the situation would change if $Z$ were increased beyond the existing periodic system. Such high- $Z$ systems can be formed for a short time in heavy-ion collisions. Here we treat the high- $Z$ system as an ordinary atom with the charge $Z=Z_{1}+Z_{2}$ being just the sum of its components. While the energy difference $E\left(1 s^{2} 2 p^{2} P_{1 / 2}\right)-E\left(1 s^{2} 2 s^{2} S_{1 / 2}\right)$ is nearly linearly increasing in the range $Z=79-94$, it again decreases in the higher $Z$ region and has a crossing point at $Z_{\text {united }} \approx 122$. This effect is due to the relativistic contraction of the $2 p_{1 / 2}$ wave function, which dominates over all other contributions for very large $Z$. For further increasing $Z$, the $2 p_{1 / 2}$ state, being below the $2 s_{1 / 2}$ state, reaches the negative energy continuum [28]. We used Desclaux's code to evaluate a number of systems for $104 \leqslant Z \leqslant 128$, with self-consistent magnetic interaction [29], vacuum polarization of order $\alpha(Z \alpha), \alpha(Z \alpha)^{3}$, and $\alpha^{2}(Z \alpha)$, self-energy extrapolated from Mohr's values and corrected for finite nuclear size. For this to be valid, however we had to limit ourselves to $Z<137$. It happens that the region of interest lies well inside this boundary. From Table $\mathrm{V}$, one can see how for such high $Z$ values the two lithiumlike states of interest cross around the united charge number $Z_{\text {united }} \approx 122$. We analyzed only symmetric collision systems, which are parity even provided their charge states are equal.

\section{POLARIZATION ROTATIONS}

This section follows the analysis given in [7] by Botz, Bruß, and Nachtmann. We follow here their notations. The energies, lifetimes, Stark and parity admixture coefficients were calculated with the multiconfiguration Dirac-Fock package from [30]. To make this paper self-contained, let us briefly repeat some of the basic arguments of [7].

The atomic system we are interested in is a lithiumlike ion that has a nonzero nuclear angular momentum. For simplicity, we take the nuclear angular momenta $I=1 / 2$ and look at the first four electron states (cf. Fig. 4). The situation for ions with other nuclear angular momentum is completely the same except that other numbers for the total angular momentum $F$ have to be inserted. (The formalism could also be applied to the boronlike case where we look at boronlike uranium-235 that has $I=7 / 2$.) The experimental situation in 
TABLE V. Energies of the first two electron states in lithiumlike heavy ions for high nuclear charges.

\begin{tabular}{|c|c|c|c|c|c|}
\hline Name & $\mathrm{Z}$ & A & $\begin{array}{l}E\left(1 s^{2} 2 s^{2} S_{1 / 2}\right) \\
\left(J^{P}=\frac{1}{2}{ }^{+}\right)(\mathrm{eV})\end{array}$ & $\begin{array}{c}E\left(1 s^{2} 2 p{ }^{2} P_{1 / 2}\right) \\
\left(J^{P}=\frac{1}{2}^{-}\right)(\mathrm{eV})\end{array}$ & $\Delta(\mathrm{eV})$ \\
\hline $\mathrm{Te}+\mathrm{Te}$ & 104 & 260 & -396234.8 & -395910.3 & 324.6 \\
\hline $\mathrm{Ce}+\mathrm{Ce}$ & 116 & 280 & -528168.1 & -527979.6 & 188.5 \\
\hline $\mathrm{Nd}+\mathrm{Nd}$ & 120 & 288 & -581273.8 & -581267.7 & 6.1 \\
\hline $\mathrm{Sm}+\mathrm{Sm}$ & 124 & 304 & -640357.1 & -640692.8 & -335.7 \\
\hline $\mathrm{Gd}+\mathrm{Gd}$ & 128 & 316 & -706756.4 & -707698.3 & -941.9 \\
\hline
\end{tabular}

which we wish to place this system is shown in Fig. 5.

The lithiumlike ion moves in the 1-direction of our coordinate system. This ion moves through alternating electric fields of width $x_{1}$, at a distance of $x_{2}$. The electric fields point in the positive and negative 3 -direction. The moving ion sees a magnetic field due to the boost, but since this field is even under parity transformation, we can neglect it. The arrangement still has one symmetry operation $\hat{R}$ under which it is invariant, and this is a combination of parity transformation and rotation about $\pi$ around the 2-axis. Together, this gives a reflection with respect to the 1-3 plane:

$$
\begin{gathered}
R:\left(\begin{array}{l}
x_{1} \\
x_{2} \\
x_{3}
\end{array}\right) \rightarrow\left(\begin{array}{c}
x_{1} \\
-x_{2} \\
x_{3}
\end{array}\right), \\
\hat{R}=e^{i \pi \hat{F}_{2}} \hat{P} .
\end{gathered}
$$

It is clear that the angular momentum states $\left|F, F_{3}\right\rangle$ are, in general, not eigenstates of this operation. But from

$$
\begin{aligned}
e^{i \pi \hat{F}_{2}}\left|F, F_{3}\right\rangle & =\sum_{F_{3}^{\prime}}\left|F, F_{3}^{\prime}\right\rangle\left\langle F, F_{3}^{\prime}\left|e^{i \pi \hat{F}_{2}}\right| F, F_{3}\right\rangle \\
& =\sum_{F_{3}^{\prime}}\left|F, F_{3}^{\prime}\right\rangle D_{F_{3}^{\prime}, F_{3}}^{(F)^{*}}(0,-\pi, 0) \\
& =\sum_{F_{3}^{\prime}}\left|F, F_{3}^{\prime}\right\rangle d_{F_{3}, F_{3}^{\prime}}^{(F)}(\pi) \\
& =\sum_{F_{3}^{\prime}}\left|F, F_{3}^{\prime}\right\rangle(-1)^{F-F_{3}^{\prime}} \delta_{F_{3}^{\prime},-F_{3}} \\
& =(-1)^{F+F_{3}}\left|F,-F_{3}\right\rangle
\end{aligned}
$$

it is easily seen that states with $F_{3}=0$ are still eigenstates of

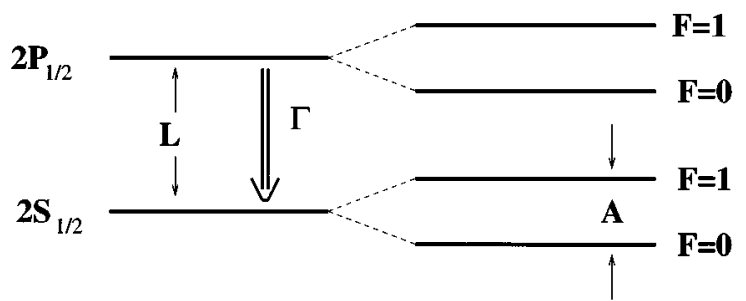

electron states hyperfine states

FIG. 4. Hyperfine splitting for the parity-mixed states. the reflection symmetry operator and, for simplicity, we will constrain our considerations to those states.

This reflection symmetry is destroyed by the weak interaction of the electron with the nucleus, which adds to the atomic Hamiltonian the terms

$$
\begin{gathered}
H_{P V}=H_{P V}^{(1)}+H_{P V}^{(2)}, \\
H_{P V}^{(1)}=-\frac{G_{F}}{\sqrt{2}} \int d^{3} x 2 g_{A}^{e} \bar{e}(\mathbf{x}) \gamma^{\lambda} \gamma_{5} e(\mathbf{x}) \\
\times\left(\sum_{q} g_{V}^{q} \bar{q}(\mathbf{x}) \gamma_{\lambda} q(\mathbf{x})\right) \\
H_{P V}^{(2)}=-\frac{G_{F}}{\sqrt{2}} \int d^{3} x 2 g_{V}^{e} \bar{e}(\mathbf{x}) \gamma^{\lambda} e(\mathbf{x}) \\
\times\left(\sum_{q} g_{A}^{q} \bar{q}(\mathbf{x}) \gamma_{\lambda} \gamma_{5} q(\mathbf{x})\right) .
\end{gathered}
$$

Here, $q$ runs over all quarks, $G_{F}$ is Fermi's constant, and $g_{A, V}^{e, q}$ denotes the neutral current coupling constants for the quark flavor $q$ or the electron $e$, respectively. Both terms together have no defined parity and consequently no defined quantum number according to the reflection symmetry operation $\hat{R}$.

On its flight the ion stays for the time $t_{1}$ in the Stark field and during the time $t_{2}-t_{1}$ outside of it. Following essentially the notation of [7], we get for the transition amplitude during the time $t_{1}$, in the case where there is no change in angular momentum,

$$
\begin{aligned}
f_{F, F_{3} ; F, F_{3}}\left(t_{1}\right)= & \exp \left\{-i E(2 \hat{S}, F) t_{1}-i \widetilde{\kappa}_{F, F_{3}}\left(\frac{\sqrt{3} \mathscr{F}}{L}\right)^{2} L t_{1}\right. \\
& \left.-\kappa_{F, F_{3}} \frac{1}{2}\left(\frac{\sqrt{3} \mathscr{F}}{L}\right)^{2} \Gamma t_{1}\right\} .
\end{aligned}
$$

In this formula we take $E(2 \hat{S}, F)$ to be the energy of the $2 S$ hyperfine states, perturbed by the parity-violating weak interaction denoted by the hat over the $S . \mathscr{F}$ is the electric stark field $\mathscr{E}$ multiplied by $e$ and the Bohr radius:

$$
\mathscr{F}=\frac{e}{Z \alpha m_{e}} \mathscr{E}
$$

$L=E_{2 S_{1 / 2}}-E_{2 P_{1 / 2}}$ is the energy difference of the two electron states of opposite parity considered in Fig. 4 and $\Gamma$ the decay 


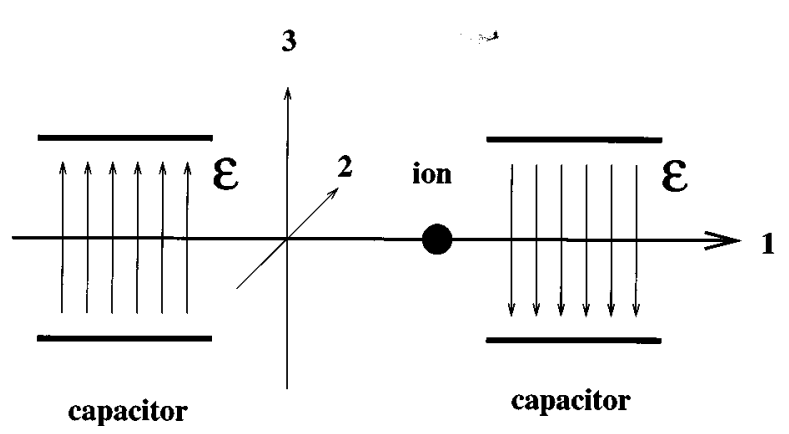

FIG. 5. Experimental setup studied for possible parity-violation measurement.

constant of the $2 P_{1 / 2}$ state mentioned above. Here, the hyperfine splitting is neglected because of its relative smallness. The $\kappa$ 's are perturbative constants that give the admixtures due to the quadratic Stark effect.

In the case where there is a transition between the angular momentum states, the amplitude is proportional to the applied electric field, i.e., e.g.,

$$
f_{1,0 ; 0,0} \sim \mathscr{F} .
$$

The total transition amplitude for an ion flying through one capacitor and the subsequent free drift length is given by

$$
g_{F^{\prime}, F_{3}^{\prime} ; F, F_{3}}=e^{-i E\left(2 \hat{S}, F^{\prime}\right)\left(t_{2}-t_{1}\right)} f_{F^{\prime}, F_{3}^{\prime} ; F, F_{3}}\left(t_{1}\right)
$$

For an experimental setup with $K$ capacitors, the amplitude for the $R$ symmetry-violating transition $\left|F=0 F_{3}=0\right\rangle$ $\rightarrow\left|F=1 F_{3}=0\right\rangle$ is

$$
\begin{aligned}
f_{1,0 ; 0,0}^{(K)} & =g_{1,0 ; 0,0} \sum_{k=0}^{K-1} g_{0,0 ; 0,0}^{k} g_{1,0 ; 1,0}^{K-k-1} \\
& =g_{1,0 ; 0,0} g_{1,0 ; 1,0}^{K-1} \frac{1-\left(\frac{g_{0,0 ; 0,0}}{g_{1,0 ; 1,0}}\right)^{K}}{1-\left(\frac{g_{0,0 ; 0,0}}{g_{1,0 ; 1,0}}\right)} .
\end{aligned}
$$

The basic idea is now to make the absolute value of such a transition amplitude large. To this end, with the definitions given before, one can express first

$$
\begin{aligned}
\frac{g_{0,0 ; 0,0}}{g_{1,0 ; 1,0}=} & \exp \left\{+i\left[A t_{2}-\left(\widetilde{\kappa}_{0,0}-\widetilde{\kappa}_{1,0}\right)\left(\frac{\sqrt{3} \mathscr{F}}{L}\right)^{2} L t_{1}\right]\right. \\
& \left.-\frac{1}{2}\left(\kappa_{0,0}-\kappa_{1,0}\right)\left(\frac{\sqrt{3} \mathscr{F}}{L}\right)^{2} \Gamma t_{1}\right\} .
\end{aligned}
$$

Here, $A=E(2 \hat{S}, 1)-E(2 \hat{S}, 0)$ denotes the energy difference due to hyperfine splitting of the $2 S$ electron orbitals. This very expression can be made real by a suitable choice of the length of the free drift space so that the condition

$$
A t_{2}-\left(\widetilde{\kappa}_{0,0}-\widetilde{\kappa}_{1,0}\right)\left(\frac{\sqrt{3} \mathscr{F}}{L}\right)^{2} L t_{1}=2 \pi n
$$

holds. We will come back to this later. With the above choice of $t_{2}$ we can get for the absolute value of the amplitude $f_{1,0 ; 0,0}^{(K)}$ the expression

$$
\left|f_{1,0 ; 0,0}^{(K)}\right| \sim\left(\sqrt{3} \mathscr{F} t_{1}\right)\left|g_{1,0 ; 1,0}\right|^{K} \frac{1-\left(\frac{g_{0,0 ; 0,0}}{g_{1,0 ; 1,0}}\right)^{K}}{1-\left(\frac{g_{0,0 ; 0,0}}{g_{1,0 ; 1,0}}\right)}=\frac{1}{2 \sqrt{Q}} .
$$

Here we have assumed $K \gg 1$. Now the aim is to maximize $\left|f_{1,0 ; 0,0}^{(K)}\right|$, which is the same as minimizing $Q$. This quantity $Q$ plays an important role in this connection because, as shown in [7], $Q$ is a measure of the polarization rotation of the ion flying through the capacitor arrangement since at $t=0$ there is no component of angular momentum $F$ parallel to the direction of flight,

$$
\left|\mathbf{e}_{\mathbf{1}} \cdot \hat{\mathbf{F}}\left(K t_{2}\right)\right| \sim \frac{1}{2 \sqrt{Q}} .
$$

For definiteness, we discuss the case of a pair of states with $F=0$ and $F=1$. We abbreviate

$$
x=\frac{1}{2}\left(\kappa_{0,0}-\kappa_{1,0}\right)\left(\frac{\sqrt{3} \mathscr{F}}{L}\right)^{2} \Gamma t_{1} K, \quad \kappa=\frac{2 \kappa_{1,0}}{\kappa_{0,0}-\kappa_{1,0}},
$$

and use as independent variables $x$ and $K$. We get up to factors independent of $K$ and $x$ :

$$
Q \sim \frac{K}{x} e^{\kappa x} \frac{\left(1-e^{-x / K}\right)^{2}}{\left(1-e^{-x}\right)^{2}} .
$$

Let us assume $K$ to be large; then $Q$ is inversely proportional to the number of capacitors $K$. We now treat $K$ as a fixed number and then look for the minimum of $Q$ as a function of $x$. As $K \gg 1$ the formal minimum of $Q$ is obtained for $x \ll 1$ such that in the vicinity of the minimum one has

$$
Q \sim \frac{e^{\kappa x}}{x K} \rightarrow x_{\min } \sim \frac{1}{\kappa} .
$$

At the minimum the quantity $\mathscr{F}$, essentially the electric field $\mathscr{E}$, is determined by

$$
\left(\frac{\sqrt{3} \mathscr{F}}{L}\right)^{2}=\frac{1}{\kappa_{1,0} K \Gamma t_{1}} .
$$

We shall discuss below that this optimal situation cannot be reached for the ions considered here. The derivation of these equations has been done for a pair of atomic states $F=0, F=1$. But there is no principal difference for other combinations such as $F=3, F=4$, which is considered here for boronlike uranium.

While the formulas are the same as those derived in [7], the quantities involved are quantitatively very different. Various large factors appear both in favor and in disfavor of the heavy-ion system and there is no simple way to estimate the relative size of the effect. We shall present the numerical results for ${ }^{235} \mathrm{U}$ in Table VI. It turns out that some light ions might also be of interest. Therefore, we also add to Table VI 
TABLE VI. Hyperfine splitting $(A)$, level width $(\Gamma)$, and level separation $(L)$ for selected ions.

\begin{tabular}{lcccc}
\hline \hline Ion & $\mathrm{Be}^{+}$ & $\mathrm{B}^{2+}$ & $\mathrm{C}^{3+}$ & $\mathrm{U}^{87+}$ \\
\hline Isotope & ${ }^{9} \mathrm{Be}$ & ${ }^{11} \mathrm{~B}$ & ${ }^{13} \mathrm{C}$ & ${ }^{235} \mathrm{U}$ \\
Lower state & $2 S_{1 / 2}$ & $2 S_{1 / 2}$ & $2 S_{1 / 2}$ & $1 s^{2} 2 s^{2} 2 p J=1 / 2$ \\
Upper state & $2 P_{1 / 2}$ & $2 P_{1 / 2}$ & $2 P_{1 / 2}$ & $1 s^{2} 2 s 2 p^{2} J=1 / 2$ \\
$\mathrm{I}$ & $3 / 2$ & $3 / 2$ & $1 / 2$ & $7 / 2$ \\
$A[\mathrm{eV}]$ & $1.71414 \times 10^{-6}$ & $1.04438 \times 10^{-5}$ & $8.44760 \times 10^{-6}$ & $1.796 \times 10^{-2}$ \\
$\Gamma[\mathrm{eV}]$ & $7.79467 \times 10^{-8}$ & $1.30629 \times 10^{-7}$ & $1.81121 \times 10^{-7}$ & $3.11949 \times 10^{-5}$ \\
$L[\mathrm{eV}]$ & $3.98910 \times 10^{+0}$ & $6.05385 \times 10^{+0}$ & $8.07181 \times 10^{+0}$ & $4.0302 \times 10^{+2}$ \\
\hline \hline
\end{tabular}

the results for the three lithiumlike systems $\mathrm{Be}^{+}, \mathrm{B}^{2+}$, and $\mathrm{C}^{3+}$. Their atomic properties are shown in Tables VII, VIII and IX. The atomic properties of ${ }^{235} \mathrm{U}$ are shown in Tables X, XI, and XII. For the calculation of the $\kappa$ coefficients we use perturbation theory:

$$
\begin{gathered}
\widetilde{\kappa}_{F, F_{3}}\left(\frac{\sqrt{3} \mathscr{F}}{L}\right)^{2} L=\sum_{n \neq m} \frac{|\langle n|e E z| m\rangle|^{2}}{E_{m}-E_{n}}, \\
\kappa_{F, F_{3}}\left(\frac{\sqrt{3} \mathscr{F}}{L}\right)^{2} \Gamma=\sum_{n \neq m}\left|\frac{\langle n|e E z| m\rangle}{E_{m}-E_{n}}\right|^{2} \Gamma_{n} .
\end{gathered}
$$

Here $m$ denotes the state with the quantum numbers $F, F_{3}$ and $n$ the other admixing states. Solving this for the $\kappa$ 's and using the Wigner-Eckart $6 j$ and $9 j$ theorems, one gets

$$
\begin{aligned}
\widetilde{\kappa}_{F, F_{3}}= & \frac{1}{3}(2 F+1) \sum_{n}\left(2 F_{n}+1\right) \frac{L}{E_{m}-E_{n}} \frac{\left|\left\langle n j_{n}|| z|| m j_{m}\right\rangle\right|^{2}}{r_{B}(Z)^{2}} \\
& \times\left(\begin{array}{ccc}
F_{n} & 1 & F \\
-F_{3} & 0 & F_{3}
\end{array}\right)^{2}\left\{\begin{array}{ccc}
F_{n} & j_{n} & I \\
j & F & 1
\end{array}\right\}^{2},
\end{aligned}
$$

$$
\begin{aligned}
\kappa_{F, F_{3}}= & \frac{1}{3}(2 F+1) \sum_{n}\left(2 F_{n}+1\right)\left(\frac{L}{E_{m}-E_{n}}\right)^{2} \\
& \times\left|\left\langle n j_{n}\|z\| m j_{m}\right\rangle\right|^{2} r_{B}(Z)^{2} \\
& \times\left(\begin{array}{ccc}
F_{n} & 1 & F \\
-F_{3} & 0 & F_{3}
\end{array}\right)^{2}\left\{\begin{array}{ccc}
F_{n} & j_{n} & I \\
j & F & 1
\end{array}\right\}^{2} .
\end{aligned}
$$

Here, $r_{B}(Z)=1 /\left(Z \alpha m_{e}\right)$. The point is now that the $\kappa$ coefficients only deviate by the small energy differences that are due to the hyperfine splitting. In Table XIII we show the $\kappa$ values for $\mathrm{Be}^{+}, \mathrm{B}^{2+}, \mathrm{C}^{3+}$, and $\mathrm{U}^{87+}$. Together with the numerical values of the hyperfine splitting and the Stark matrix elements, which are given in Tables VII-XII, we can calculate the expressions of interest for the polarization rotation effects. Let us first start in the same way as in [7] and analyze the situation for the minimal $Q$. It turns out that this assumption would imply unrealistically large electrical fields resulting from

$$
t_{2}=\frac{\widetilde{\kappa}_{0,0}-\widetilde{\kappa}_{1,0}}{\kappa_{1,0}} \frac{L}{\Gamma A}\left[\frac{\hbar}{e}\right] \frac{1}{K} \quad(n=0) .
$$

TABLE VII. Atomic structure for lithiumlike ${ }^{9} \mathrm{Be}$.

\begin{tabular}{lcc}
\hline \hline $2 p_{1 / 2}-2 s_{1 / 2}$ PNC matrix element & & $-5.6810174 \times 10^{-15} \mathrm{eV}$ \\
$2 p_{1 / 2}-2 s_{1 / 2}$ energy difference & & $3.9891026 \times 10^{+00} \mathrm{eV}$ \\
$2 p_{1 / 2}-2 s_{1 / 2}$ lifetime (length) & & $8.4443912 \times 10^{-09} \mathrm{~s}$ \\
$2 p_{1 / 2}-2 s_{1 / 2}$ lifetime (velocity) & & $7.9463913 \times 10^{-09} \mathrm{~s}$ \\
$2 p_{1 / 2}-2 s_{1 / 2}$ Stark element & & $0.76491 \times 10^{+00} \mathrm{a} . \mathrm{u}$. \\
\hline $2 p_{1 / 2} F=2$ & total hyperfine matrix element: & $-1.1844518555 \times 10^{-07} \mathrm{eV}$ \\
& Bohr-Weisskopf correction: & $5.9033102475 \times 10^{-15} \mathrm{eV}$ \\
& total: & $-1.1844517964 \times 10^{-07} \mathrm{eV}$ \\
& & \\
$2 p_{1 / 2} F=1$ & total hyperfine matrix element: & $1.9740864258 \times 10^{-07} \mathrm{eV}$ \\
& Bohr-Weisskopf correction: & $-9.8388504126 \times 10^{-15} \mathrm{eV}$ \\
& total: & $1.9740863274 \times 10^{-07} \mathrm{eV}$ \\
$2 s_{1 / 2} F=2$ & & \\
& total hyperfine matrix element: & $-6.4289041847 \times 10^{-07} \mathrm{eV}$ \\
& Bohr-Weisskopf correction: & $8.8568061986 \times 10^{-11} \mathrm{eV}$ \\
& total: & $-6.4280185040 \times 10^{-07} \mathrm{eV}$ \\
$2 s_{1 / 2} F=1$ & & \\
& total hyperfine matrix element: & $1.0714840308 \times 10^{-06} \mathrm{eV}$ \\
& Bohr-Weisskopf correction: & $-1.4761343664 \times 10^{-10} \mathrm{eV}$ \\
& total: & $1.0713364173 \times 10^{-06} \mathrm{eV}$ \\
& &
\end{tabular}


TABLE VIII. Atomic structure for lithiumlike ${ }^{11} \mathrm{~B}$.

\begin{tabular}{llc}
\hline \hline $2 p_{1 / 2}-2 s_{1 / 2}$ PNC matrix element & & $-2.7316505 \times 10^{-14} \mathrm{eV}$ \\
$2 p_{1 / 2}-2 s_{1 / 2}$ energy difference & & $6.0538537 \times 10^{+00} \mathrm{eV}$ \\
$2 p_{1 / 2}-2 s_{1 / 2}$ lifetime (length) & & $5.0387842 \times 10^{-09} \mathrm{~s}$ \\
$2 p_{1 / 2}-2 s_{1 / 2}$ lifetime (velocity) & & $4.6813678 \times 10^{-09} \mathrm{~s}$ \\
$2 p_{1 / 2}-2 s_{1 / 2}$ Stark element & & $.52970 \times 10^{+00} \mathrm{a} . \mathrm{u}$. \\
\hline $2 p_{1 / 2} F=2$ & total hyperfine matrix element: & $8.7104273520 \times 10^{-07} \mathrm{eV}$ \\
& Bohr-Weisskopf correction: & $-8.9772008878 \times 10^{-14} \mathrm{eV}$ \\
& total: & $8.7104264543 \times 10^{-07} \mathrm{eV}$ \\
& & \\
$2 p_{1 / 2} F=1$ & total hyperfine matrix element: & $-1.4517378920 \times 10^{-06} \mathrm{eV}$ \\
& Bohr-Weisskopf correction: & $1.4962001480 \times 10^{-13} \mathrm{eV}$ \\
& total: & $-1.4517377424 \times 10^{-06} \mathrm{eV}$ \\
& & \\
$2 s_{1 / 2} F=2$ & total hyperfine matrix element: & $3.9170586600 \times 10^{-06} \mathrm{eV}$ \\
& Bohr-Weisskopf correction: & $-6.4395425787 \times 10^{-10} \mathrm{eV}$ \\
& total: & $3.9164147057 \times 10^{-06} \mathrm{eV}$ \\
& & \\
$2 s_{1 / 2} F=1$ & total hyperfine matrix element: & $-6.5284311000 \times 10^{-06} \mathrm{eV}$ \\
& Bohr-Weisskopf correction: & $1.0732570965 \times 10^{-09} \mathrm{eV}$ \\
& total: & $-6.5273578429 \times 10^{-06} \mathrm{eV}$ \\
\hline \hline
\end{tabular}

From (12) we get the requirement for the individual effects to add:

$$
\left[A t_{2}-\frac{\widetilde{\kappa}_{0,0}-\widetilde{\kappa}_{1,0}}{\kappa_{1,0}} \frac{L}{K \Gamma}\right]=2 \pi n
$$

This implies that the deviation $\delta t_{2}$ in $t_{2}$ should be smaller than

$$
\delta t_{2}<\frac{1}{100} \frac{1}{A}\left[\frac{\hbar}{e}\right]
$$

For the time $t_{1}$, which gives the length of the capacitor, we are required to take $t_{1} \leqslant t_{2}$, but there are no other constraints. To make the required electric field small, one has to choose $t_{1}$ large [see Eq. (25) below], so we take $t_{1}=t_{2} / 2$. Finally, from the relation

$$
\left(\frac{\sqrt{3} \mathscr{F}}{L}\right)^{2}=\frac{1}{\kappa_{1,0} \Gamma t_{1}}\left[\frac{\hbar}{e}\right] \frac{1}{K}
$$

one can well calculate the electric field. The terms in $[\cdots]$ always give the necessary factors for the translation into SI

TABLE IX. Atomic structure for lithiumlike ${ }^{13} \mathrm{C}$.

\begin{tabular}{lcc}
\hline \hline $2 p_{1 / 2}-2 s_{1 / 2}$ PNC matrix element & $-8.7153650 \times 10^{-14} \mathrm{eV}$ \\
$2 p_{1 / 2}-2 s_{1 / 2}$ energy difference & $8.0718138 \times 10^{+00} \mathrm{eV}$ \\
$2 p_{1 / 2}-2 s_{1 / 2}$ lifetime (length) & $3.6341015 \times 10^{-09} \mathrm{~s}$ \\
$2 p_{1 / 2}-2 s_{1 / 2}$ lifetime (velocity) & $3.3515826 \times 10^{-09} \mathrm{~s}$ \\
$2 p_{1 / 2}-2 s_{1 / 2}$ Stark element & & $.40514 \times 10^{+00} \mathrm{a} . \mathrm{u}$. \\
\hline $2 p_{1 / 2} F=0$ & total hyperfine matrix element: & $-1.5529296692 \times 10^{-06} \mathrm{eV}$ \\
& Bohr-Weisskopf correction: & $3.0240473822 \times 10^{-13} \mathrm{eV}$ \\
& total: & $-1.5529293668 \times 10^{-06} \mathrm{eV}$ \\
& total hyperfine matrix element: & $5.1764322307 \times 10^{-07} \mathrm{eV}$ \\
$2 p_{1 / 2} F=1$ & Bohr-Weisskopf correction: & $-1.0080157941 \times 10^{-13} \mathrm{eV}$ \\
& total: & $5.1764312227 \times 10^{-07} \mathrm{eV}$ \\
& total hyperfine matrix element: & $-6.3369865130 \times 10^{-06} \mathrm{eV}$ \\
$2 s_{1 / 2} F=0$ & Bohr-Weisskopf correction: & $1.2851809538 \times 10^{-09} \mathrm{eV}$ \\
& total: & $-6.3357013320 \times 10^{-06} \mathrm{eV}$ \\
& Bohr-Weisskopf correction: & $-4.2839365127 \times 10^{-10} \mathrm{eV}$ \\
& total: & $2.1119004440 \times 10^{-06} \mathrm{eV}$ \\
\hline \hline
\end{tabular}


TABLE X. Atomic level structure for boronlike ${ }^{235} \mathrm{U}$.

\begin{tabular}{lcc}
\hline \hline Level & $\begin{array}{c}\text { Binding } \\
\text { energy } \\
(\mathrm{eV})\end{array}$ & $\begin{array}{c}\text { Excitation } \\
\text { energy } \\
(\mathrm{eV})\end{array}$ \\
\hline ground state & -358233.01 & \\
$1 s^{2} 2 s 2 p^{2} J=1 / 2$ & -357829.98 & 403.02 \\
$1 s^{2} 2 s 2 p^{2} J=3 / 2$ & -353861.72 & 4371.29 \\
$1 s^{2} 2 s 2 p^{2} J=5 / 2$ & -353818.09 & 4414.92 \\
$1 s^{2} 2 s^{2} 2 p J=3 / 2$ & -354139.11 & 4093.90 \\
$1 s^{2} 2 s 2 p^{2} J=1 / 2$ & -353712.14 & 4520.87 \\
\hline \hline
\end{tabular}

TABLE XI. $1 s^{2} 2 s^{2} 2 p J=\frac{1}{2}-1 s^{2} 2 s 2 p^{2} J=\frac{1}{2}$ matrix elements in boronlike ${ }^{235} \mathrm{U}$.

\begin{tabular}{lc}
\hline \hline PNC matrix element & $3.79 \times 10^{-06} \mathrm{eV}$ \\
Lifetime velocity gauge & $3.06 \times 10^{-11} \mathrm{~s}$ \\
Lifetime length gauge & $2.11 \times 10^{-11} \mathrm{~s}$ \\
Stark element & $0.29048 \times 10^{-03}$ a.u.
\end{tabular}

PNC matrix element

Lifetime length gauge

Stark element

TABLE XII. Hyperfine structure in boronlike ${ }^{235} \mathrm{U}$.

\begin{tabular}{|c|c|c|c|}
\hline $1 s^{2} 2 s^{2} 2 p$ & $j=1 / 2, F=3, I=7 / 2$ & $\begin{array}{l}\text { hyperfine: } \\
\text { Bohr-Weisskopf: } \\
\text { total: }\end{array}$ & $\begin{array}{c}1.02 \times 10^{-02} \mathrm{eV} \\
-1.14 \times 10^{-04} \mathrm{eV} \\
1.01 \times 10^{-02} \mathrm{eV}\end{array}$ \\
\hline $1 s^{2} 2 s^{2} 2 p$ & $j=1 / 2, F=4, I=7 / 2$ & $\begin{array}{l}\text { hyperfine: } \\
\text { Bohr-Weisskopf: } \\
\text { total: }\end{array}$ & $\begin{array}{c}-7.95 \times 10^{-03} \mathrm{eV} \\
8.83 \times 10^{-05} \mathrm{eV} \\
-7.86 \times 10^{-03} \mathrm{eV}\end{array}$ \\
\hline $1 s^{2} 2 s 2 p^{2}$ & $j=1 / 2, F=3, I=7 / 2$ & $\begin{array}{l}\text { hyperfine: } \\
\text { Bohr-Weisskopf : } \\
\text { total: }\end{array}$ & $\begin{array}{c}3.03 \times 10^{-02} \mathrm{eV} \\
-9.83 \times 10^{-04} \mathrm{eV} \\
2.94 \times 10^{-02} \mathrm{eV}\end{array}$ \\
\hline $1 s^{2} 2 s 2 p^{2}$ & $j=1 / 2, F=4, I=7 / 2$ & $\begin{array}{l}\text { hyperfine: } \\
\text { Bohr-Weisskopf : } \\
\text { total: }\end{array}$ & $\begin{array}{c}-2.36 \times 10^{-02} \mathrm{eV} \\
7.65 \times 10^{-04} \mathrm{eV} \\
-2.28 \times 10^{-02} \mathrm{eV}\end{array}$ \\
\hline
\end{tabular}

TABLE XIII. $\kappa$ values.

\begin{tabular}{lcc}
\hline \hline Ion & $\kappa$ constants & Numerical values \\
\hline $\mathrm{Be}^{+}$ & $\kappa_{1,0}, \widetilde{\kappa}_{1,0}$ & \multicolumn{1}{c}{$\kappa_{2,0}, \widetilde{\kappa}_{2,0}$} \\
& $\kappa_{1,0}, \widetilde{\kappa}_{1,0}$ & $5.2007794253 \times 10^{-1} ;-5.2007778741 \times 10^{-1}$ \\
$\kappa_{2,0}, \widetilde{\kappa}_{2,0}$ & $5.2007741321 \times 10^{-1} ;-5.2007752275 \times 10^{-1}$ \\
$\mathrm{~B}^{2+}$ & & \\
& $\kappa_{0,0}, \widetilde{\kappa}_{0,0}$ & $3.8969640353 \times 10^{-1} ;-3.8969687978 \times 10^{-1}$ \\
$\mathrm{C}^{3+}$ & $3.8969804714 \times 10^{-1} ;-3.8969770158 \times 10^{-1}$ \\
& & \\
$\mathrm{U}^{87+}, \widetilde{\kappa}_{1,0}$ & $3.2827626620 \times 10^{-1} ;-3.2827654492 \times 10^{-1}$ \\
& $\kappa_{3,0}, \widetilde{\kappa}_{3,0}$ & $3.2827712174 \times 10^{-1} ;-3.2827697269 \times 10^{-1}$ \\
& $\kappa_{4,0}, \widetilde{\kappa}_{4,0}$ & $3.9683205819 \times 10^{-5} ;-3.9679966597 \times 10^{-5}$ \\
\hline \hline
\end{tabular}

TABLE XIV. Characteristic values for selected ions.

\begin{tabular}{lcccc}
\hline \hline Ion & $\mathrm{Be}^{+}$ & $\mathrm{B}^{2+}$ & $\mathrm{C}^{3+}$ & $\mathrm{U}^{87+}$ \\
\hline$t_{2}[s]$ & $1.0000 \times 10^{-08}$ & $6.1594 \times 10^{-09}$ & $4.5248 \times 10^{-09}$ & $8.2441 \times 10^{-11}$ \\
$\delta t_{2}[s]$ & $3.8399 \times 10^{-12}$ & $6.3024 \times 10^{-13}$ & $7.7917 \times 10^{-13}$ & $3.6649 \times 10^{-16}$ \\
$t_{1}$ & $5.0000 \times 10^{-09}$ & $3.0797 \times 10^{-09}$ & $2.2624 \times 10^{-09}$ & $4.1221 \times 10^{-11}$ \\
$x$ & $5.0888 \times 10^{-07}$ & $-2.1088 \times 10^{-06}$ & $-1.3031 \times 10^{-06}$ & $1.7412 \times 10^{-04}$ \\
$Q_{\min }$ & $2.2789 \times 10^{-16}$ & $2.0174 \times 10^{-16}$ & $1.8042 \times 10^{-16}$ & $8.2689 \times 10^{-20}$ \\
$\mathscr{E}\left[\frac{\mathrm{V}}{\mathrm{m}}\right]$ & $3.1371 \times 10^{+11}$ & $6.7668 \times 10^{+11}$ & $1.1688 \times 10^{+12}$ & $4.5952 \times 10^{+16}$ \\
\hline \hline
\end{tabular}


TABLE XV. $x$ and $Q$ for realistic values of $K, \mathscr{E}$, and $t_{1}$.

\begin{tabular}{lcccc}
\hline \hline Ion & $\mathrm{Be}^{+}$ & $\mathrm{B}^{2+}$ & $\mathrm{C}^{3+}$ & $\mathrm{U}^{87+}$ \\
\hline$x$ & $1.0341 \times 10^{-20}$ & $-1.4954 \times 10^{-20}$ & $-4.2159 \times 10^{-21}$ & $2.0004 \times 10^{-26}$ \\
$Q$ & $2.0629 \times 10^{-06}$ & $3.2232 \times 10^{-06}$ & $4.6414 \times 10^{-06}$ & $1.0912 \times 10^{-03}$ \\
\hline \hline
\end{tabular}

units. The resulting numbers are given in Table XIV. Here we always set $K=1$. The values for other $K$ can easily be determined from the formulas above. Note that $K$ has to be chosen very large and that the electric field $\mathscr{E}$ is, for the choice $t_{1}=t_{2} / 2$ or for any choice $t_{1} \sim t_{2}$, independent of $K$. Table XIV shows the results for $\mathrm{Be}^{+}, \mathrm{B}^{2+}, \mathrm{C}^{3+}$, and $\mathrm{U}^{87+}$. The values for the electric field $\mathscr{E}$ are so unrealistically large that such an experiment cannot be realized. The reason for the large values of $\mathscr{E}$ is the fact that in atoms with more than one electron the energy difference between the $2 p_{1 / 2}$ and the $2 s_{1 / 2}$ states is orders of magnitude larger than for hydrogenlike atoms because the $2 s_{1 / 2}-2 p_{1 / 2}$ degeneracy is eliminated by the electron-electron interaction.

We now proceed in the opposite direction. We take a realistic field $\mathscr{E}$ and other realistic values

$$
K=1000, \quad \mathscr{E}=1000 \frac{V}{m}, \quad t_{1}=1.0 \times 10^{-8} \mathrm{~s}
$$

We then calculate

$$
x=\frac{1}{2}\left(\kappa_{0,0}-\kappa_{1,0}\right)\left(\frac{\sqrt{3} \mathscr{F}}{L}\right)^{2} \Gamma t_{1} K\left[\frac{e}{\hbar}\right] .
$$

As $x$ is very small, we approximate

$$
\begin{aligned}
Q= & \frac{\left(\kappa_{0,0}-\kappa_{1,0}\right) \Gamma K}{8 L^{2} t_{1}} \frac{1}{x}\left(1-e^{\left.-\frac{x}{K}\right)^{2}\left(1-e^{-x}\right)^{-2}}\right. \\
& \times \exp \left(\frac{2 \kappa_{1,0}}{\kappa_{0,0}-\kappa_{1,0}} x\right) \rightarrow \frac{\kappa_{0,0}-\kappa_{1,0}}{8 L^{2} t_{1}} \Gamma \frac{1}{x K}\left[\frac{\hbar}{e}\right] .
\end{aligned}
$$

In this way we get the values of Table XV. These values must be compared to that obtained in [7] for hydrogen $Q_{\min }=6.6 \times 10^{-9}$.

\section{CONCLUSIONS}

In principle, it is obvious that heavy ions with few inner shell electrons offer a possibility of testing the effects of parity admixture. This admixture is, in heavy ions, orders of magnitude larger than in neutral atoms.

The ideal case is one in which parity-violation has a sizable effect without applying any of the elaborate methods used in the cesium experiment [3]. Then, the only chance of getting measurable parity admixtures lies in finding a pair of energy states near the ground state with equal angular momenta but opposite parity that is nearly degenerated with respect to its energy. Unfortunately, there is no such pair of orbitals in uranium with 2-5 electrons except for the already known degeneracy in heliumlike uranium. As the electron levels only change very slowly with $Z$, the same is true for the neighboring heavy ions.

The next step will consequently be a very detailed analysis of the degeneracy in heliumlike heavy ions including nuclear and isotopic effects because here a level crossing must exist. Level crossing also exists for compound nuclear reactions but here the lifetime of the compound nucleus is too short to allow for atomic physics experiments. Looking for parity-violating spin rotations opened another perspective. We showed, however, that the net effect (value of $1 / \sqrt{Q})$ for heavy ions is about thirty times weaker than for hydrogen.
[1] C. Bouchiat, C. A. Piketty, and D. Pignon, Nucl. Phys. B 221, 68 (1983).

[2] C. Bouchiat and C. A. Piketty, Phys. Lett. B 128, 73 (1983).

[3] M. C. Noecker, B. P. Masterson, and C. E. Wieman, Phys. Rev. Lett. 61, 310 (1988).

[4] W. J. Marciano and A. Sirlin, Phys. Rev. D 27, 552 (1983).

[5] J. L. Rosner, Phys. Rev. D 53, 2724 (1996).

[6] A. Schäfer, G. Soff, P. Indelicato, B. Müller, and W. Greiner, Phys. Rev. A 40, 7362 (1989).

[7] G. W. Botz, D. Bruß, and O. Nachtmann, Ann. Phys. 240, 107 (1995).

[8] U. Feldman, J. Sugar, and P. Indelicato, J. Opt. Soc. Am. B 8, 3 (1991).

[9] P. Indelicato, Phys. Scr. (to be published).

[10] F. Parente, J. P. Marques, and P. Indelicato, Europhys. Lett. 26, 437 (1994).

[11] I. P. Grant et al., Comput. Phys. Commun. 21, 207 (1980).

[12] D. R. Plante, W. R. Johnson, and J. Sapirstein, Phys. Rev. A 49, 3519 (1994).
[13] P. Indelicato, O. Gorceix, and J. P. Desclaux, J. Phys. B 20, 651 (1987).

[14] G. W. F. Drake, Can. J. Phys. 66, 586 (1988).

[15] P. Indelicato and L. De Billy (private communication).

[16] P. Indelicato, Phys. Rev. A 51, 1132 (1995).

[17] P. J. Mohr, and G. Soff, Phys. Rev. Lett. 70, 158 (1993).

[18] P. Indelicato and P. J. Mohr, Theor. Chem. Acta 80, 207 (1991).

[19] S. A. Blundell, Phys. Scr. T46, 144 (1993).

[20] S. A. Blundell, P. J. Mohr, W. R. Johnson, and J. Sapirstein, Phys. Rev. A 48, 2615 (1993).

[21] S. A. Blundell, W. R. Johnson, and J. Sapirstein, Phys. Rev. A 41, 1698 (1990).

[22] A. Ynnerman, J. James, I. Lindgren, H. Persson, and S. Salomonson, Phys. Rev. A 50, 4671 (1994).

[23] W. R. Johnson, J. Sapirstein, and K. T. Cheng, Phys. Rev. A 51, 297 (1995).

[24] Y. K. Kim, D. H. Baik, P. Indelicato, and J. P. Desclaux, Phys. Rev. A 44, 148 (1991). 
[25] P. Indelicato and J. P. Desclaux, Phys. Rev. A 42, 5139 (1990).

[26] W. R. Johnson, S. Blundell, and J. Sapirstein, Phys. Rev. A 37, 2764 (1988).

[27] J. P. Marques, F. Parente, and P. Indelicato, Phys. Rev. A 47, 929 (1993).
[28] W. Greiner, B. Müller, and J. Rafelski, Quantum Electrodynamics of Strong Fields (Springer, Berlin, 1985).

[29] P. Indelicato, J. Phys. B 19, 1719 (1986).

[30] J. P. Desclaux, in Methods and Techniques in Computational Chemistry, edited by E. Clementi (STEF, Cagliari, 1993), Vol. A. 